\title{
Apoio Matricial: um estudo bibliográfico
}

\author{
Matrix Support: a bibliographical study
}

Alexandra Iglesias ${ }^{1}$

Luziane Zacché Avellar ${ }^{2}$
${ }^{1}$ Programa de Pós-

Graduação em Psicologia,

Universidade Federal do

Espírito Santo. Av. Fernando

Ferrari 514/Prédio Lídio de

Souza/sala 6, Goiabeiras.

29.075-910 Vitória ES

Brasil.leiglesias@gmail.com

${ }^{2}$ Departamento de

Psicologia Social e do

Desenvolvimento, Programa

de Pós-Graduação em

Psicologia, Universidade

Federal do Espírito Santo.
Abstract This article presents a bibliographical review of matrix support in mental health. A search was conducted in the Virtual Health Library and the LILACS, SciELO and Google Scholar databases using the key words: "matrix support in mental health." Fourteen articles were located with the desired characteristics, which indicates that only a restricted number of publications are in circulation. The articles were analyzed with respect to their structural and methodological aspects, which revealed the absolute predominance of the use of qualitative methods and health professionals as the target research population. The same articles were then analyzed for their theoretical discussions. Among other issues, the importance of matrix support to enhance the primary health care teams provided to people suffering from psychic distress is highlighted. However, there is still considerable confusion regarding the proposal of the matrix support and shared responsibilities between teams of reference and mental health professionals, which emphasizes the need for training of these professionals, as well as better coordination and organization of the mental health care network.

Key words Matrix support, Mental health, Bibliographical review, Primary health care
Resumo Este artigo constitui-se de um estudo de revisão bibliográfica a respeito do apoio matricial em saúde mental. Foi realizada uma busca nas bases de dados da Biblioteca Virtual da Saúde Lilacs e SciELO - e do Google acadêmico, com as palavras-chave "apoio matricial em saúde mental" elou "matriciamento em saúde mental". Foram encontrados 14 artigos com as características desejadas, o que evidencia o número restrito de publicações sobre o tema. Os artigos foram analisados quanto a sua parte estrutural e metodológica, destacando a predominância absoluta do uso do método qualitativo e dos profissionais de saúde como população alvo das pesquisas. Em seguida, estes mesmos artigos foram analisados quanto a suas discussões teóricas. Ressalta-se, dentre outras questões, a importância do apoio matricial para potencialização das equipes da atenção básica no cuidado às pessoas em sofrimento psíquico. Contudo, ainda são muitas as confusões quanto à proposta do apoio matricial e as responsabilidades compartilhadas entre equipes de referências e profissionais de saúde mental, o que apontam para a necessidade de capacitação destes profissionais, além de uma melhor articulação e organização da rede de cuidado em saúde mental.

Palavras-chave Apoio matricial, Saúde mental, Revisão bibliográfica, Atenção básica 


\section{Introdução}

Por um longo período na história, a hospitalização, o enclausuramento, a exclusão e o isolamento foram tomados como única forma de lidar com a "loucura". As pessoas em sofrimento psíquico, "desprovidas" de sua potência produtiva, em período de crescimento e fortalecimento do capitalismo, eram depositadas nos manicômios, onde sofriam com a violação explícita de sua integridade física, moral, psíquica. Até que na década de 80 , frente ao contexto de movimentação política e social para a democratização do Brasil, esse modelo hospitalocêntrico e medicalizante passou a ser fortemente criticado, favorecendo a reestruturação do modelo assistencial à saúde mental, preconizado pelo Movimento da Reforma Psiquiátrica.

Tal movimento tem como foco a desinstitucionalização, que não se restringe a desospitalização, já que a retirada destas pessoas dos hospitais não acarreta numa mudança automática das atitudes de intolerância frente à diferença. A desinstitucionalização implica em mudança cultural, política, social, ideológica e ética no modo de lidar com o sofrimento psíquico. Para tanto, requer a criação de condições para que estas pessoas recém saídas dos hospitais psiquiátricos, e os novos pacientes, sejam inseridos socialmente, acolhidos e tratados em seu sofrimento.

Neste contexto, a articulação da Saúde Mental e da Atenção Básica torna-se imprescindível, considerando a Unidade de Saúde (US) como espaço mais próximo à comunidade local, onde as pessoas em sofrimento vivem e devem ser inseridas socialmente. A Unidade de Saúde, como representante da atenção básica, figura como porta de entrada do setor, o primeiro nível de contato da população com o sistema nacional de saúde, onde as pessoas chegam com as mais variadas queixas e devem ser acolhidas em suas questões de saúde, por mais que este acompanhamento não se restrinja a este espaço. Esta proximidade com a comunidade pode favorecer a construção de uma nova lógica de relação e acolhimento às pessoas em sofrimento psíquico pela sociedade como um todo.

Delgado $^{1}$ chama a atenção para a obrigatoriedade desta interface da saúde mental com as outras situações cotidianas das Unidades de Saúde, bem como as potencialidades desta articulação, na medida em que o usuário passa a ser acolhido em sua integralidade, reconhecido os fatores sociais, políticos, econômicos, culturais e subjetivos como determinantes e condicionantes do proces- so saúde/doença, no entendimento do Ministério da Saúde².

Existe um componente de sofrimento subjetivo associado a toda e qualquer doença, às vezes atuando como entrave à adesão a práticas preventivas ou de vida mais saudáveis. Poderíamos dizer que todo problema de saúde é também - e sempre - de saúde mental, e que toda saúde mental é também - e sempre-produção de saúde. Nesse sentido, será sempre importante e necessária a articulação da saúde mental com a Atenção Básica².

Neste contexto, surge em 2003 a proposta do apoio matricial, que tem por objetivo contribuir para o acolhimento das pessoas em sofrimento mental nos espaços sociais onde circula, por meio de uma construção coletiva de saberes junto às equipes de saúde da família ${ }^{3}$. Trata-se de uma proposta recente, daí o interesse e objetivo deste trabalho de conhecer o que vem sendo produzido teoricamente sobre o assunto, que possa dar sustentação a outras construções sobre o apoio matricial em saúde mental.

O Apoio Matricial se constituiu como proposta do Ministério da Saúde para a articulação entre a rede de Saúde Mental e as Unidades de Saúde, com vistas à implementação de uma clínica ampliada, ao compartilhamento no cuidado a estes usuários, à integração dialógica entre diferentes categorias profissionais e especialidades, à promoção à saúde e à disponibilização de outras ofertas terapêuticas através de um profissional de saúde mental que acompanhe sistematicamente as USs.

Em outras palavras, o apoio matricial em saúde mental tem por função dar suporte, discutir, intervir conjuntamente e capacitar os profissionais das Unidades de Saúde no cuidado à saúde mental, promovendo a corresponsabilização dos casos existentes e aumentando a capacidade resolutiva das equipes de referência no manejo com esses usuários ${ }^{4}$.

Trata-se de assegurar retaguarda assistencial e suporte técnico-pedagógico às equipes que prestam atenção aos problemas de saúde da população. Neste processo, as equipes de referência - lotadas nas USs - atuam como responsáveis pela condução do caso ao longo do tempo, enquanto o apoiador matricial (como especialista que é), podendo estar lotado nos CAPSs, objetiva contribuir na composição de propostas de intervenção para uma situação de saúde específica a ser resolvida ${ }^{5}$.

$\mathrm{Na}$ proposta de Apoio Matricial existem, necessariamente, estas duas equipes - uma de referência e outra de apoiadores - que devem 
compartilhar experiências, saberes e poderes para a tomada de decisões, igualmente, de modo integrado. A equipe de referência é responsável pela condução do caso, com vistas à ampliação das possibilidades de vínculo favorável no tratamento e avanço da intervenção junto ao caso em discussão. $\mathrm{O}$ apoiador matricial, por sua vez, é um especialista que pode agregar um conhecimento, contribuindo para a intervenção pensada na resolução de determinado problema de saúde.

$O$ fato de serem especialistas não significa que detenham a resposta para a resolução do caso, a ideia é a construção compartilhada de possibilidades de intervenção para a situação em pauta, como traz Dimenstein et al. ${ }^{6}$, somente a integralidade pode ser realmente efetiva na promoção à saúde. Neste sentido, o apoio requer um trabalho interdisciplinar e encontros sistemáticos entre as duas equipes, para a troca de saberes na construção de projetos terapêuticos, bem como para a definição de responsabilidades específicas para cada ator envolvido neste trabalho.

Dito isto, o Apoio Matricial se afirma como uma metodologia de trabalho potente na estruturação da rede de saúde por meio da transformação das relações entre os profissionais e, consequentemente, destes com os outros atores sociais, incluindo os usuários. É a partir das relações que o sujeito, o mundo e a própria saúde se constituem cotidianamente. Como afirma Campos $^{7}$, uma relação de poder altamente verticalizada e centralizada em suas decisões tende ao descompromisso e alienação dos atores da saúde.

Todos têm a contribuir na resolução dos problemas de saúde, integrando atenção e cuidado às pessoas. No caso do Apoio Matricial, o apoiador procura contribuir de modo compartilhado os projetos de intervenção com a equipe de referência, considerando o seu conhecimento específico, sua experiência, sua visão de mundo e de sujeito, bem como as demandas dos outros profissionais, com seus conhecimentos, desejos, interesses e visão de mundo e de sujeito.

Assim, é possível desbancar as fragmentações alimentadas pela crescente especialização das várias áreas do conhecimento e promover alterações na subjetividade e na cultura dominante do campo da saúde, que passarão a centrar-se no diálogo, na integralidade, no trabalho em equipe, na corresponsabilização e na escuta qualificada.

Diante da relevância da proposta do apoio matricial, faz-se importante conhecer o que já foi produzido sobre este assunto, bem como os métodos utilizados para se chegar às discussões suscitadas pelo tema em questão. Certamente, o estudo de revisão bibliográfica possibilita o desenho de outros trabalhos sobre este objeto, a partir das verificações, análises e comparações das informações colhidas.

\section{Método}

O presente trabalho constitui-se em um estudo de revisão bibliográfica à respeito do apoio matricial em saúde mental. A escolha por este modo de estudar o matriciamento vem da intenção de conhecer o que vem sendo produzido sobre o tema, as discussões suscitadas e as metodologias utilizadas nos artigos científicos referentes ao assunto. Para tanto, foi realizada uma busca nas bases de dados da Biblioteca Virtual da Saúde - Lilacs e SciELO - e do Google acadêmico, no período de abril a julho de 2011, utilizando das seguintes palavras-chave: "apoio matricial em saúde mental” e/ou "matriciamento em saúde mental" (entendendo apoio matricial e matriciamento como palavras sinônimas). No caso do Google acadêmico, utilizamos sua pesquisa avançada, indicando que o artigo encontrado deveria conter a frase exata: "apoio matricial em saúde mental" e/ou "matriciamento em saúde mental", em qualquer lugar do texto. Nas outras bases de dados, apenas inserimos as palavras-chave mencionadas para a busca dos artigos indexados.

Foram encontrados 14 artigos com as características desejadas. As teses de doutorado encontradas e outros trabalhos, como de conclusão de curso, por exemplo, foram excluídos, já que a proposta era analisar apenas artigos sobre o tema, publicados em periódicos científicos e indexados em bases de dados de fácil acesso.

Após o levantamento dos artigos, foi construída uma ficha para anotação das informações contidas nos textos, que auxiliaria o pesquisador na análise posterior dos artigos levantados. Tal ficha constava do nome do artigo, objetivo, delineamento metodológico, técnicas de coleta, local de estudo, população, técnica de análise, principais resultados, periódico e ano de publicação.

Para a análise dos artigos, utilizamos uma leitura exploratória e analítica a fim de alcançar os objetivos deste estudo. Primeiramente, no item resultados e discussões deste artigo, apresentaremos as questões referentes à parte estrutural e metodológica dos artigos analisados e, em seguida, traremos as discussões teóricas, obtidas pela análise dos resultados destes mesmos artigos. 


\section{Resultados e discussões}

\section{Parte estrutural e metodológica dos artigos}

Foram encontrados 14 artigos, distribuídos em onze diferentes revistas nas áreas de Saúde Coletiva (9), Saúde Mental (2), Psicologia (2) e Enfermagem (1), publicados nos anos de 2007 a 2010, todos em idioma português, o que se relaciona ao fato do apoio matricial tratar-se de uma proposta nacional. Os textos com os quais trabalhamos têm como local de estudo o sul, sudeste e nordeste do país, nenhum artigo foi encontrado oriundo do centro-oeste e do norte do Brasil.

O Apoio Matricial pode ser considerado uma proposta recente, descrita inicialmente por Cam$\operatorname{pos}^{7}$, em 1999, como um arranjo organizacional para o trabalho. Seguiu-se com a sua implantação em alguns serviços de saúde de Campinas e, só então, em 2003, foi adotado pelo Ministério da Saúde, para a articulação da rede de saúde mental com a atenção primária à saúde, o que justifica o fato dos artigos encontrados serem de datas atuais (2007 a 2010).

Todos os artigos levantados foram delineados pela metodologia qualitativa, abrangendo relatos de experiências, pesquisa de campo e textos teóricos. Em relação aos artigos que utilizaram de pesquisa de campo, todos tiveram como população alvo os profissionais de saúde, utilizando-se, como técnica de coleta de dados, a observação, entrevista semi-estruturada, questionários, grupos focais, documentos oficiais, reuniões, discussões e visitas domiciliares. Neste último caso, com registros fonográfico, fotográfico e em relatório. Para análise destes dados, os artigos contaram com a técnica de análise de conteúdo, análise descritiva, análise comparativa e meta-narrativa. Vale destacar que alguns deles não especificaram a técnica de análise utilizada.

A análise de conteúdo tem sua maior expressão em Bardin ${ }^{8}$, cuja obra apresenta em detalhes a fundamentação teórica da técnica e seu modo de aplicação. A técnica de análise de conteúdo tem por objetivo "obter, por procedimentos, sistemáticos e objetivos de descrição do conteúdo das mensagens, indicadores (quantitativos ou não) que permitam a inferência de conhecimentos relativos às condições de produção/recepção (variáveis inferidas) destas mensagens".

A análise de conteúdo requer um trabalho de leitura e releitura do material coletado, seguida da categorização e interpretação dos dados. Os artigos listados para esta revisão, que se utilizaram da análise de conteúdo, buscaram avaliar as informações quanto aos seus conteúdos, categorizando-os.

Outra técnica utilizada foi a análise descritiva, que na explicação dos autores dos artigos consiste "na operação de codificação, classificação e categorização dos dados"9. Neste sentido, os dois artigos ${ }^{9,10}$ que empregaram tal modo de análise também elaboraram categorias para apresentação e discussão dos dados, assemelhando-se à análise de conteúdo. Contudo, diferencia-se por assumir uma forma aproximada ao do levantamento, o qual se encarrega de descrever as características do fenômeno por meio de técnicas mais padronizadas, como o questionário (como foi o caso de um dos artigos) ou a entrevista direta com a pessoa de interesse para a pesquisa ${ }^{11}$. As categorias construídas por estes dois artigos, para a análise descritiva, se assemelham, em seu conteúdo, às descritas nos outros artigos analisados, na medida em que buscam descrever o conhecimento/percepção dos profissionais sobre os transtornos mentais mais frequentes, entendimentos sobre o apoio matricial e as práticas junto às pessoas em sofrimento psíquico.

Também foi utilizado para análise, em um dos artigos listados nesta revisão, o método comparativo constante ${ }^{12}$, que consiste na identificação das unidades de informação - sentenças obtidas do material empírico, compreensível a qualquer leitor - e na categorização - cujo objetivo é unificar todas as unidades de informação relacionadas ao mesmo conteúdo -, para então traçar as semelhanças e diferenças entre o material levantado na pesquisa.

A metanarrativa ${ }^{13}$, utilizada em outro artigo, versou sobre a interpretação das narrativas trazidas pelos profissionais - público-alvo - nos grupos focais.

Ainda em relação aos métodos utilizados nos artigos, chama atenção o fato de todos os textos empregarem a metodologia qualitativa, o que para Minayo ${ }^{14}$ demonstraria uma intenção de se trabalhar com o universo de significados, motivações, aspirações, crenças, valores e atitudes dos processos em questão. Diante da situação de todos os artigos utilizarem o método qualitativo, sentimos a necessidade de trazer para esta revisão uma discussão sobre a pesquisa qualitativa e quantitativa, não que qualquer dos artigos professe a primazia de um ou outro método, mas vale destacar a possibilidade de analisar o tema de modos e enfoques diferentes e o quanto isto pode ser enriquecedor para se pensar uma determinada realidade social. Como nos traz Minayo ${ }^{14}$, os fenômenos sociais podem ser analisados nas suas 
relações, histórias e representações, mas também nas suas regularidades e frequências. Entendemos que tal discussão pode contribuir para se pensar em outros modos de se pesquisar o apoio matricial em saúde mental, que enfoque também as propriedades numéricas do fenômeno (tão valorizadas para o financiamento das ações em saúde), para além de suas qualidades.

Discute-se que, por muito tempo, predominou a quantificação para a legitimação científica. Neste sentido, o que era produzido fora desta lógica de mensuração não tinha o valor científico. Neste contexto, outro movimento de afirmação da importância e valor da pesquisa qualitativa se estruturou no cenário científico, mas muito no sentido de negação do outro método, enquanto o argumento deveria ser no sentido de valorização das duas abordagens, que igualmente possuem vantagens e desvantagens, pontos positivos e negativos, cada uma tem seu papel, seu lugar e sua adequação, dependendo prioritariamente da pergunta de pesquisa, que, por sua vez, pode ser multifacetada, comportando mais de um método ${ }^{14,15}$.

No setor saúde, assim como em outras áreas, a possibilidade de contribuição da interação dos dois métodos e de suas teorias para a análise das questões referentes a este campo está justamente em suas diferenças, e quando trabalhadas em conjunto (e não em oposição) podem promover uma "mais elaborada e completa construção da realidade, ensejando o desenvolvimento de teorias e de novas técnicas cooperativas" ${ }^{\prime 4}$. Isto para o apoio matricial, certamente, pode ter uma potencialidade/contribuição importante, uma vez que se trata de uma prática incipiente, ainda em processo de construção. Pensar, por exemplo, indicadores, que tomados como parte da qualidade do apoio matricial, possam dar maior sustentação a esta prática inovadora, que tem por desafio promover mudanças nas práticas hegemônicas da saúde, potencializando o papel da intersetorialidade e da integralidade no cuidado à pessoa em sofrimento psíquico na atenção básica.

Por fim, em relação à metodologia dos artigos, deve ser destacado o fato de que todos eles têm como público-alvo os profissionais de saúde. Os objetivos foram diversos: uns trouxeram a proposta de avaliar o apoio matricial e de discutir a implantação desta estratégia, outros visaram o relato de experiência e a análise da participação do psicólogo na efetivação do apoio, teve ainda o artigo que buscou identificar em documentos oficiais em que medida e de que forma as políticas públicas contemplavam a atuação do psicólogo na atenção básica, incluindo a prática do apoio matricial em saúde mental. Independente dos objetivos, todos os artigos que se utilizaram de uma população para a coleta de dados e não de documentos, focaram no ponto de vista dos profissionais em relação à proposta do apoio. Mas, qual seria a avaliação dos usuários e das famílias que compartilham desta prática?

O SUS traz em seu bojo, assim como as políticas que lhe dão sustentação, a valorização de todos os atores sociais (usuários, gestores e trabalhadores), no processo de efetivação das práticas em saúde. Daí podermos afirmar da importância de se explorar as repercussões do apoio matricial nas vidas e no cuidado à saúde destas pessoas que usufruem desta nova prática antimanicomial.

\section{Discussões teóricas trazidas pelos artigos}

Dentre os resultados dos artigos levantados foi presença marcante a avaliação, pelos profissionais, da importância do apoio matricial para potencialização das equipes das Unidades de Saúde no cuidado às pessoas em sofrimento psíquico ${ }^{9,10,12,13,16-19}$. Inclusive um dos artigos chega a pontuar a diminuição do estresse das equipes, uma vez que conseguem compartilhar responsabilidades dos casos mais complexos com outros setores da saúde ${ }^{20}$.

Neste sentido, as equipes conseguem trabalhar uma das prioridades da Estratégia de Saúde da Família, a Promoção à Saúde, que traz justamente como um de seus pilares a intersetorialidade e a integralidade do cuidado, valorizando sobremaneira o trabalho em equipe. Associado a isto, as intervenções tendem a se tornar mais efetivas do ponto de vista terapêutico, uma vez que a Unidade de Saúde, como porta de entrada do sistema de saúde, revela-se mais propícia à construção de vínculo entre profissional-usuário, indispensável à efetivação de espaços coletivos de luta por melhorias nas condições gerais de vida destas pessoas ${ }^{10}$.

O entendimento de alguns profissionais é de que o acompanhamento do usuário deve ser feito na Unidade de Saúde, próximo à sua família e a sua comunidade ${ }^{12}$, local propício a se trabalhar a reinserção social e a desinstitucionalização, conforme preconizada pela política nacional de saúde mental. Isto significa trabalhar o cuidado a esta pessoa em sofrimento e a comunidade como um todo, para a mudança de uma mentalidade que, por muito tempo, construiu-se pautada na ideia de que a pessoa com transtorno mental deveria ser mantida reclusa, já que necessariamente representava perigo à população. 
A estratégia do Apoio Matricial surge, justamente, a partir da defesa de que a pessoa em sofrimento mental deve ser acolhida em seu território de origem, integrada à rede social, a qual deve fazer parte, a fim de que a promoção à saúde aconteça. No plano internacional, o ponta-pé inicial foi dado pela Declaração de Caracas, que em 1990 propôs a reestruturação da atenção às pessoas em sofrimento por meio da vinculação saúde mental e atenção básica. No Brasil, a movimentação pelo cuidado destas pessoas, também na atenção básica, ganha força a partir de 2001, quando a Coordenação Nacional de Saúde Mental promove a "Discussão do Plano Nacional de Inclusão das Ações de Saúde Mental na Atenção Básica”, seguido de um Seminário sobre o tema em 2002, a integração do assunto ao sétimo Congresso Brasileiro de Saúde Coletiva em 2003 e, no mesmo ano, a edição da circular "Saúde Mental na Atenção Básica: o vínculo e o diálogo necessários - Inclusão das ações de Saúde Mental na Atenção Básica", que traz a proposta do Apoio Matricial da Saúde Mental às equipes da Atenção Básica, o modo de se organizar as ações de apoio e as responsabilidades compartilhadas entre as equipes matriciais de Saúde Mental e Atenção Básicaa ${ }^{21}$.

Contudo, no dia-a-dia ainda há confusões em relação à proposta do apoio matricial ${ }^{6} \mathrm{e}$ às responsabilidades compartilhadas entre equipes de referências e os profissionais de saúde mental, os quais, muitas vezes, depositam as dificuldades de efetivação do apoio matricial na resistência, que percebem, das equipes de referências de trabalharem com o que não é medicamentoso, ou ainda por sentirem que as equipes pensam a proposta como uma imposição da gestão. Neste sentido, os profissionais de saúde mental têm dificuldades em reconhecer seus próprios limites em compartilhar saberes e responsabilidades no cuidado às pessoas em sofrimento mental ${ }^{13}$.

As equipes de referências, por sua vez, neste mesmo artigo ${ }^{13}$, também no sentido de transferir responsabilidades, descrevem o "apoio que dá certo" e o que "não dá certo". Referem o "apoio que dá certo" como aquele em que conseguem encaminhar o caso aos apoiadores de modo que estes o absorvam para si.

Já o "apoio que não dá certo" é descrito como aquele em que a equipe de referência é convocada a discutir o caso, em que o apoiador se coloca a ajudar a equipe a acompanhar o caso; ou seja, o que é considerado "insucesso" ocorre quando o apoiador não assume sozinho o encaminhamento da situação em questão. Desta forma, o cuidado à demanda de saúde mental é encarado como mais uma questão para a atenção básica dar conta e como uma desresponsabilização dos serviços especializados 6

A efetividade e a eficácia do apoio matricial em saúde mental dependem de um trabalho conjunto. Portanto, não se trata de eleger responsáveis pelos entraves à efetivação do apoio, mas o reconhecimento da complexidade envolta à sua concretização, para que então as dificuldades sejam trabalhadas com vistas à maior resolutividade da atenção.

Foi descrito por vários profissionais, em especial aqueles que compõem as equipes de referência, a necessidade de uma capacitação específica para se atender aos usuários de saúde mental $^{4,6,10,13}$. Há que se considerar que trabalhar com esta clientela é desafiador, pela história estigmatizante que carregam e pelas angústias possivelmente despertadas nos profissionais no contato com o sofrimento. Tanto que a inserção do psicólogo nas equipes de apoiadores matriciais em saúde mental tem sido uma indicação inclusive por algumas políticas públicas ${ }^{22,23}$.

Faz-se importante, portanto, a presença do apoiador junto a estas equipes de referência para auxiliá-las no sentido de desmistificar alguns entendimentos a respeito da loucura, desfazendo preconceitos que, algumas vezes, resultam na segregação das pessoas em sofrimento psíquico no próprio espaço de atendimento à saúde.

Fora do campo 'psi', ainda são poucas as formações acadêmicas que abordam o tema da saúde mental, o que representa um entrave à intervenção das demais categorias junto a estas pessoas. Neste sentido, os artigos ${ }^{4,6,10,13}$ demonstram que, muitas vezes, a participação dos vários profissionais que compõem à Unidade de Saúde no cuidado às pessoas em sofrimento, ainda depende da disponibilidade profissional, do seu perfil ou da sua formação complementar.

Sendo assim, o apoio matricial mais uma vez afirma sua importância, uma vez que tem também a função de suporte técnico-pedagógico a estas equipes de referência, possibilitando que mais profissionais estejam habilitados a intervir junto às pessoas em sofrimento menta ${ }^{17}$, fazendo valer os princípios da universalidade de acesso, integralidade e equidade da atenção, por exemplo.

A proposta traz em seu bojo a intenção de qualificar os profissionais no lidar com complexidade da vida de cada sujeito, independente de uma patologia. Há que se trabalhar pela Promoção à Saúde e pela Prevenção de Doenças com vistas à construção de um sistema de saúde de qualidade, considerando as dimensões socioculturais, 
políticas, econômicas, biopsicossociais e familiares que cotidianamente constroem os sujeitos.

Figueiredo e Campos ${ }^{24}$ destacam o papel do apoio matricial em saúde mental de ofertar aos profissionais de saúde, de um modo geral, conhecimentos e ações (historicamente considerados próprios das áreas 'psi') que necessariamente irão auxiliar estes profissionais na ampliação da escuta e consequentemente da clínica, do acolher e do lidar com as subjetividades presentes no espaço da Unidade. Vale pontuar que não se trata de rejeitar as especificidades da área, mas de participar com este saber na promoção coletiva de outros e novos saberes que possibilitem uma atenção mais efetiva. Isto remete à superação da lógica de fragmentação e especialização do trabalho, que se materializa em inúmeros encaminhamentos do usuário de um local a outro sem a menor interconexão e conhecimento, por parte dos profissionais envolvidos, das intervenções pensadas e realizadas no cuidado ao caso.

É indispensável o fortalecimento da rede de atenção, de modo que os serviços estejam articulados em suas ações e mantenham uma relação direta com vistas à integralidade do cuidado. Assim, o apoio rejeita a ideia de encaminhamentos, trabalha com a construção de projetos terapêuticos singulares para cada sujeito, de acordo com suas necessidades, de modo que a responsabilidade para sua concretização seja compartilhada com todos os atores sociais, transcendendo o setor saúde, já que toma a saúde em seu conceito ampliado, envolve as condições gerais de vida (lazer, educação, saneamento básico, alimentação etc.), como determinantes à saúde.

A necessidade de articulação e organização da rede de cuidado em saúde mental comparece como mais uma questão a ser trabalhada conjuntamente para a efetivação deste apoio. Os artigos ${ }^{4,6,12,24}$ apontam o imperativo da oferta de outros equipamentos de acolhimento destas pessoas para a garantia do acesso a um acompanhamento terapêutico adequado e de qualidade.

De acordo com dados do Ministério da Saú$\mathrm{de}^{25}$, cerca de $20 \%$ da população brasileira necessitaria de algum cuidado em saúde mental, sendo $3 \%$ pessoas que vivem com transtorno mental severo e persistente e que, portanto, deveriam receber cuidados contínuos. Os transtornos menos graves representam $9 \%$ da população, demandando cuidados eventuais, e outros $8 \%$ apresentam transtornos decorrentes do uso prejudicial de álcool e outras drogas, necessitando de atendimento regular. Isto representa, aproximadamente, 38 milhões de pessoas, o que certamente sig- nifica uma demanda elevada de necessidade de cuidado e que, muitas vezes, tem deixado de ser acolhida, mesmo após a implantação do apoio, pelas dificuldades presentes na estruturação da rede de saúde.

Dimenstein et al. ${ }^{6}$ trazem que ainda são pequenas as possibilidades de referenciamento destas pessoas em sofrimento, tendo em vista a precariedade da rede de serviços substitutivos e da articulação destes com os demais serviços do SUS. Há que se ofertar outros e novos equipamentos $^{12}$, bem como potencializar a implantação de CAPSIII, de leitos em hospitais gerais, de centros de convivência e cultura, de serviços ambulatoriais de saúde mental, de equipes da ESF na atenção a estas pessoas, a fim de que se possa oferecer um cuidado integral e de qualidade.

\section{Considerações finais}

O apoio matricial tem se apresentado como uma possibilidade real de conversão de um contexto de exclusão das pessoas em sofrimento, tanto que sua contribuição pela efetivação da promoção à saúde é destacada em todos os artigos analisados para este estudo. Isto porque tem conseguido levar à atenção básica a discussão da inclusão da saúde mental em suas práticas de cuidado.

Contudo, é certo que se tem muito a avançar. Por meio deste estudo pudemos apreender que há uma carência de trabalhos que explorem, por exemplo, as contribuições de usuários e familiares para esta prática inovadora. Certamente, como um dos principais afetados por esta prática, usuários e familiares têm a acrescentar em avaliações que venham enriquecer este novo modo de se acolher o sofrimento psíquico.

Trata-se de uma proposta em construção, razão pela qual reforçamos a importância e necessidade de outros estudos que trabalhem outros métodos de investigação, para além da exclusividade do método qualitativo. Destacamos, também, a importância de pesquisas que explorem as possibilidades de avaliação desta prática e definam mais claramente algumas responsabilidades para cada um dos atores envolvidos.

Por fim, com base nos artigos analisados nesta revisão, indicamos o investimento em capacitações para os profissionais e o fortalecimento da rede de cuidado como imprescindível para que o tratamento das pessoas em sofrimento psíquico esteja garantido fora da ideia de exclusão, pautado na atenção psicossocial na comunidade onde habita. 


\section{Colaboradores}

\author{
A Iglesias e LZ Avellar participaram igualmente \\ de todas as etapas de elaboração do artigo.
}

\section{Referências}

1. Delgado PGG. Política Nacional de Saúde Mental: O Contexto decisivo do Pacto pela Saúde. Jornal do CONASEMS 2007; (ago):8-9.

2. Brasil. Ministério da Saúde. Reforma Psiquiátrica e Política de Saúde Mental no Brasil - Conferência Regional de Reforma dos Serviços de Saúde Mental: 15 anos Depois de Caracas. Brasília, 07 a 10 de novembro de 2005.

3. Campos FCB, Nascimento SPS. O apoio matricial: reciclando a saúde mental na atenção básica. Cad IPUB 2007; 13(24):57-66.

4. Bezerra E, Dimenstein M. Os CAPS e o trabalho em rede: tecendo o apoio matricial na atenção básica. Psicol Cienc Prof 2008; 28(3):632-645.

5. Campos GWS, Domitti AC. Apoio matricial e equipe de referência: uma metodologia para gestão do trabalho interdisciplinar em saúde. Cad Saude Publica 2007; 23(2):399-407.

6. Dimenstein M, Severo AK, Brito M, Pimenta AL, Medeiros V, Bezerra E. O apoio matricial em Unidades de Saúde da Família: experimentando inovações em saúde mental. Saúde Soc 2009; 18(1):63-74.

7. Campos GWS. Equipes de referência e apoio especializado matricial: um ensaio sobre a reorganização do trabalho em saúde. Cien Saude Colet 1999; 4(2):393-403.

8. Bardin L. Análise de conteúdo. Lisboa: Edições 70; 1988.

9. Barreto M, Foppa AA, Cabral MF, Gonçalves JR, More CLOO. Acolhimento em Saúde Mental: percepções de profissionais vinculados à Estratégia de Saúde da Família. Rev Tempus Actas Saúde Colet 2010; 4(1):39-49.

10. Harada OL, Soares MH. A percepção do agente comunitário de saúde para identificar a depressão. SMAD Rev. Eletrônica Saúde Mental Álcool Drogas 2010; 6(2): 315-336.

11. Gil AC. Como elaborar projetos de pesquisa. São Paulo: Atlas; 1991.

12. Mielke FB, Olchowsky A. Saúde mental na Estratégia Saúde da Família: a avaliação de apoio matricial. SMAD Rev. Eletrônica Saúde Mental Álcool Drogas 2010; 63(6):900-907.

13. Figueiredo MD, Campos RO. Saúde Mental na atenção básica à saúde de Campinas, SP: uma rede ou um emaranhado? Cien Saude Colet 2009; 14(1):129-138.

14. Minayo MCS. O Desafio do Conhecimento: Pesquisa Qualitativa em saúde. 9a ed. revista e aprimorada. São Paulo: Hucitec; 2006.

15. Günther H. Pesquisa Qualitativa Versus Pesquisa Quantitativa: Esta É a Questão? Psicologia: Teoria e Pesquisa 2006; 22(2):201-210.
16. Carneiro AC, Oliveira ACM, Santos MMS, Alves MS, Casais NA, Santos JE. Saúde mental e atenção primária: uma experiência com agentes comunitários de saúde em Salvador-BA. Rev. Brasileira em Promoção da Saúde. 2009; 22(4):264-271.

17. Arona EC. Implantação do matriciamento nos serviços de saúde de Capivari. Saúde Soc 2009; 18(1): 26-36.

18. Soares MH. A inserção do enfermeiro psiquiátrico na equipe de apoio matricial em saúde mental SMAD Rev. Eletrônica Saúde Mental Álcool Drogas 2008; 4(2):1-12.

19. Tófoli LF, Fortes S. O apoio matricial de saúde mental na atenção primária no município de Sobral, CE: o relato de uma experiência. Sanare 2007; 6(2):34-42.

20. Barban EG, Oliveira AA. O modelo de assistência da equipe matricial de saúde mental no Programa Saúde da Família do município de São José do Rio Preto. Arq Cienc Saude 2007; 14(1):54-65.

21. Gama CAP, Onocko-Campos R. Saúde mental na atenção básica - uma pesquisa bibliográfica exploratória em periódicos de saúde coletiva (1997-2007). Cad Bras Saúde Mental 2009; 1(2):112-131.

22. Ferreira Neto JL. Psicologia e saúde mental: três momentos de uma história. Saude Debate 2008; 32(78):1826.

23. Boing El, Crepaldi MA. O Psicólogo na atenção básica:: uma incursão pelas políticas públicas de saúde Brasileiras. Psicol Cienc Prof 2010; 30(3):634-649.

24. Figueiredo MD, Campos RO. Saúde Mental e Atenção Básica à Saúde: o apoio matricial na construção de uma rede multicêntrica. Saúde Debate 2009; 32(79):143-149.

25. Brasil. Ministério da Saúde (MS). Saúde Mental e Atenção Básica: o vínculo e o diálogo necessários. In: Brasil. Ministério da Saúde. Saúde Mental no SUS: os centros de atenção psicossocial. Brasília: MS; 2003. p. 77-84.

Artigo apresentado em 15/03/2013

Aprovado em 23/06/2013

Versão final apresentada em 15/08/2013 